Everitt L, Fenwick J, Homer CSE. (2015). Midwives experiences of removal of a newborn baby in New South Wales, Australia: Being in the head and heart space. Women and Birth 28 (2):95-100.

\title{
Midwives experiences of removal of a newborn baby in New South Wales, Australia: Being in the head and heart space
}

\begin{abstract}
Background: A newborn baby is removed from his or her mother into formal care when he/she is considered at risk of serious harm and it is not in the best interests to go home with their parent(s) or carer(s). In New South Wales (NSW), this removal is known as an "assumption of care". This process is challenging for all involved especially when it occurs soon after birth. There is very limited research to inform midwives in this area of practice.
\end{abstract}

Aim: To explore the experiences of midwives who had been involved in the assumption of care of a baby soon after birth or in the early postnatal period.

Method: A qualitative descriptive approach was used. Ten midwives involved with the assumption of care of a baby were interviewed. A thematic analysis was undertaken.

Findings: There were two overarching themes. "Being in the head space" represented the activities, tasks and/or processes midwives engaged in when involved in an assumption of care. "Being in the heart space" described the emotional impact on midwives, as well as their perceptions on how women were affected.

Conclusion: Midwives described feeling unprepared and unsupported, in both the processes and the impact of assumption of care. They were confronted by this profound emotional work and described experiencing professional grief, similar to that felt when caring for a woman experiencing a stillbirth. In the future, midwives need to be provided with support to ensure that they can effectively care for these women and also manage the emotional impact themselves. 


\section{INTRODUCTION}

Being separated from her baby soon after birth is one of the most traumatic events that can happen to a woman who has just given birth. A forced separation may occur if there are concerns about the safety of the baby. In New South Wales (NSW), Australia, this process of separation and removal is known as an "assumption of care" 1 .

In NSW, the Children and Young Persons (Care and Protection) Legislation Act 1998, defines when an assumption of care of a child or young person can occur. A policy based on the Act states that:

An assumption of care order may be issued where Community Services suspects, on reasonable grounds, that the child or young person is at risk of serious harm and is satisfied that it is not in the best interests of the child or young person to be removed from the Health premises by their parent(s)/carer(s). In these circumstances the Chief Executive Community Services may assume the care responsibility of a child or young person by means of an order in writing served on the person who can reasonably be assumed to be in charge of the Health premises at the time. ${ }^{1}$

The policy provides instructions on the procedures and process of assumption of care, however, they are silent on how best to assist the health care professionals through this event ${ }^{2}$.

As primary providers of antenatal care, midwives have a unique opportunity to recognise vulnerable families during pregnancy, optimise health and wellbeing and reduce risk of serious harm to the unborn child. ${ }^{3}$ If little or no improvements occur during pregnancy and there is evidence of risk of serious harm to an infant, the midwife as a mandatory reporter is obliged to notify the authorities. If a rapid response is required, the Department of Community Services (DoCS) in NSW applies for an order to 'assume care and responsibility' for the newborn immediately post birth ${ }^{2}$. In 2009, the Department of Community Services (DoCS) in NSW changed its name to Community Services and in this paper is known as 'Community Services'. 
There is little research investigating assumption of care from the perspective of midwives. An extensive search of the literature identified only one relevant study, a qualitative study of nine midwives in the United Kingdom (UK). ${ }^{4}$ The midwives recognised the important, but difficult, part they played in child protection including a lack of appropriate collaboration, communication and support. The midwives also reported feeling scared and fearful as a result of the family's anger at the removal of a newborn and made comparisons between the grief and loss reactions of these women and those seen after a stillbirth. The research concluded that it was important to find ways to better understand these experiences and support midwives during these rare but emotionally distressing situations.

Other research, ${ }^{5,6}$ highlights how the removal of a newborn is one of the most forceful interventions of the State into a family's life. This study showed that the tension between a "woman centred" versus "child/fetus focus" approach, potentially causing internal conflict for the midwife and heightening feeling of powerless and vulnerability.

These two studies highlight how assumption of care is challenging for midwives but it is not known if this is similar in Australia. Our study aimed to explore and describe midwives' experiences of being involved in the care of a woman at risk of an assumption of care and/or whose newborn had been removed. The study was approved by the university's human research ethics committee prior to commencement (HREC No: $2010-223 A$ ).

\section{METHOD}

A qualitative descriptive design was used to describe the phenomena of being involved in the assumption of care. ${ }^{7,8}$ Descriptive studies enable a rich description of complex situations that are unexplored in the literature and search for a deeper understanding of the experience of participants ${ }^{9}$. Data were collected using in-depth interviews. The analysis aimed to produce a 
comprehensive description of how midwives perceived and experienced the events surrounding "assumption of care".

\section{Recruitment of participants}

Midwives working in metropolitan Sydney (NSW) who had been involved with an assumption of care within the past three years were invited to participate. The timeframe was to ensure that participants had practised during the most recent legislative changes surrounding child protection. A half-page advertisement was placed in the Australian College of Midwives (ACM) - NSW Branch newsletter. A number of midwives contacted the research team after hearing about the study from their colleagues ${ }^{8}$. Upon contact, midwives were given detailed information about the study and what participation would involve. Interested midwives, who met the inclusion criteria, were sent an information sheet and consent form. Any midwife who identified herself/himself as a work colleague of the first author was excluded to avoid potential conflict of interest during the interview process.

\section{Participant characteristics}

Ten midwives, aged between 40-59 years with 2-30 years of experience as a midwife, were interviewed. All participants were female. Nine were midwives with a prior nursing qualification and one was a graduate from a direct-entry Bachelor of Midwifery program. All had completed some type of continuing education qualification. The participants worked in a range of models of care including rotating through all areas of maternity care, providing caseload care or as managers or clinical midwifery consultants. Eight worked in metropolitan public hospitals and two in rural public hospitals. The midwives had been directly involved in between one to 18 assumptions of care in the past three years.

\section{Data Collection}


Prior to the interview, a brief overview of the interview question guide was forwarded to the participant. Each individual interview was held at a convenient time and in a location of the participants choosing. Signed consent forms were collected at the commencement of the interview.

Each participant was interviewed once. Interviews lasted between 45 and 60 minutes, were digitally recorded and transcribed verbatim by the first author. While the interview guide included a broad set of promoting questions the sequence remained flexibility to ensure the researcher could appropriately respond to participants (see Box 1). As the analysis progressed, concepts related to the emerging themes were added. Field notes were made before and after each interview. Theoretical saturation was reached after nine interviews. ${ }^{10,}{ }^{11}$ One additional midwife was interviewed to verify saturation.

\section{Data Analysis}

Thematic analysis was used to identify, explore and report themes within the text. ${ }^{12}$ The process outlined by Burns and Grove ${ }^{12}$ was used to guide the analysis process. Transcribed interviews were read numerous times in combination with the field notes. Initial thoughts were documented. Lineby-line coding then commenced which identified the main concepts. ${ }^{13}$ These concepts were allocated codes which were clustered into groups and given tentative labels in an iterative manner. Eventually, themes and/or sub themes were formed from the grouped data. Relationships and links between themes were explored. ${ }^{12}$ An audit trail was kept of the analytical decisions to ensure clarity and transparency of the analysis process. ${ }^{13-15}$

During the analysis phase, the preliminary findings were presented at a number of midwifery and maternity conferences. These presentations provided additional opportunities to reflect on the concepts and the meanings associated with the emerging themes ${ }^{13 .}$

\section{FINDINGS}




\section{Themes}

Two major themes were identified. "Being in the headspace" represented the activities, tasks and/or processes that midwives engaged in when involved in an assumption of care. "Being in the heart space" described the emotional impact on midwives, as well as their perceptions on how women were affected.

\section{"Being in the head space"}

"Being in the head space" was used by a number of the midwives to describe how they "mentally" separated their feelings or emotions from the process and procedures they needed to undertake when providing care to a woman who was at risk of and/ or experienced an assumption of care. In essence, it represented the intellectual work of the assumption of care. This captured the midwife's knowledge and understandings of the complexities, the importance of child protection procedures as well as the work they undertook in trying to prevent an assumption of care. Appreciating and coming to terms with the circumstances in which the newborn needed protection meant that some midwives could rationalise the assumption of care. The following quote from one midwife reflects how many of the participants felt:

Most of the time, I can resolve it in my head and can make sense of it. It doesn't mean that you don't feel for the mother, but you know that the baby is probably better off not in that environment (Midwife 8).

The more experience that a midwife had with the procedural process of an assumption of care event, which included working with Community Services antenatally, the better prepared she felt to support women and ultimately maintain child safety. The following interview extract sums up "being in the headspace": 
The head space is just about knowing that you've done everything that you can. That the woman's challenges or issues have been identified early, that you've put stuff into place, that you've got a team together to try and support them, and that it's not just an automatic..."you're losing the baby"'. So that's the headspace, that you've done everything that you can while protecting the baby's safety (Midwife 5).

"Being in the headspace" also meant learning to work with Community Services to ensure the best outcome for the woman and her baby. Midwives used a number of strategies to either, work with women to have the best chance of keeping their baby or prepare them for their removal. For example, this midwife suggested:

You've got to work with Community Services, not against them. Everything that they are asking the woman to do, every hoop that they put there, you've got to help her jump through it. Really encouraging them to engage and sometimes we have had really lovely outcomes where at the beginning of the pregnancy you thought there was no way will they keep the baby, and women have actually come through in the end. They did everything that Community Services have asked them and it's been a really good outcome (Midwife 9).

Midwives recognised the need to "walk a fine line" or "do a dance" when reporting to Community Services. While on the whole, involving the woman was considered the best approach, at times midwives felt that they could not let a woman know about a report to Community Services. For example:

Sometimes you know that a report has gone in, you might have done it yourself. If I made the report the majority of the time I will tell the woman. There is the occasion that I don't because I am really concerned that she will disengage (Midwife 8). 
Establishing and maintaining a positive relationship with the woman was one way to maintain engagement and ensure she had access to resources that could assist her turn her situation around. Midwives considered it was important to give women "hope". As one said:

You're trying to engage and trying to make a plan and trying to do everything possible to see that with enough support these parents can take their child home and care for them safely, which is what midwifery is supposed to be about (Midwife 5).

The complexity of the cases involving vulnerable women and their babies meant that planning took time. As one midwife commented: "hours and hours and hours of meetings and planning and liaising and one woman can probably take your whole day". Management plans ensured that the staff would know what was going to happen if Community Services assumed care of the baby. "Being all on the same page" was particularly important because an assumption of care may occur at any time:

It was all in place, the staff knew what the process was to follow, the after-hours hospital managers knew what was to happen, the postnatal ward knew. So everybody was filled in, briefed on what was to happen (Midwife 6).

While midwives did not always agree with the decision being made to assume care of the baby, they recognised that some babies "should just not go home with their mother". Parents with serious mental health issues or significant drug and alcohol problems were common examples. However, midwives still struggled with the assumption of care, saying:

In my head I know that it's for the best reason for the child, for the safety of the child but there is still a woman who has had a baby removed, a woman with dreams (Midwife 4).

There were examples where "being in the head space" required midwives to "remain silent" and not disclose that Community Services had decided to remove the baby after birth. In most circumstances 
this was a consequence of having a genuine concern that the mother or family would abscond with the baby soon after birth. At times safety of staff was also a consideration:

In some cases, we would have been advised by Community Services because of safety issues for the child and the staff, either from the woman or her partner or family, not to inform them, and so we're very careful not let them see the notification (Midwife 5).

For midwives working from a position of logic also meant adopting strategies to make the process and procedure of assumption as smooth as possible. This included knowing when an assumption of care might occur; creating a safe place for the removal; being there when the woman or family was "served with the court order"; being present for the woman; and only "involving security staff and police when absolutely necessary". Some midwives also verbalised that they preferred that social worker to actually remove the baby as this reduced their sense of role conflict.

In summary, midwives used their head or their intellectual knowledge and understanding to come to terms with the assumption of care. Engaging women in this way helped midwives to stay focused on delivering the different aspects of care woman required despite the potentially distressing situation.

\section{"Being in the heart space"}

"Being in the heart space" was the overarching theme that captured the emotional impact of being involved with an assumption of care. In this space, midwives were working from their 'heart' recognising that any woman facing the removal of her baby was likely to be "anxious", "scared" and "fearful".

Midwives used the words "crying", "distress", "defeated", "shock" and "devastation" to describe women's reactions to the news that Community Services were assuming care of their baby. More disturbing, were the vocal responses from the women at the actual time of removal. One midwife said she cared for a woman who was "literally clawing at the door with blood curdling screams". 
Midwives rarely forgot witnessing this level of emotional distress stating how the images "stuck in their memories".

Midwives described how many women also displayed "fury", "anger" and "aggression". They shared numerous stories of the intense rage that some women and families demonstrated before, during and after the assumption of care. This could be directed at Community Services workers, social workers, security staff or themselves. As one midwife shared; "She tore out of the room with security chasing her and she was just like a wild animal. Hell hath no fury like somebody trying to take a mother's cub, all that anthropological stuff makes perfect sense" (Midwife 7).

Despite the overt anger, midwives recognised these were normal and justified responses. As one midwife said; "If I took notice of everything they say to you I wouldn't come to work. And I think it is just the emotion, they're upset you're taking their baby away" (Midwife 6).

In some situations, once the baby had been removed, the midwives described how grief would overcome the woman. Midwives talked about women "losing hope" of ever being able to have their baby returned or even have access visits, for example saying:

"The mum has given up hope and doesn't feel it is even worth pursuing. She feels that she will never have her children back' (Midwife 1).

"Her initial reaction when they took the baby away was "OK, that's it; I'm going to give up. I'm not going to go and see the babies at all anymore" (Midwife 9).

The requirement to report a woman whose baby was assessed to be at risk was also difficult. While midwives could rationalise the need to prioritise the baby it was, none-the-less, a heart-wrenching decision that challenged their notion of partnership and woman centred care. One midwife explained: 
Because it was quite a different role for midwifery, because midwifery is about supporting women, being with women, woman centred ... but for this population you often became, you are the voice of the baby, because the baby has no voice and you need to be there for the baby (Midwife 7).

Midwives shared the "emotional rollercoaster" with women as they struggled to come to terms with shifting their focus from 'being with woman' to protecting the baby. Commonly the only way midwives could explain their emotional responses was to relate or compare the experience to caring for a woman who had a stillborn baby. For example one said, "It is that complete raw emotional pain that you are subjected to... you see it sometimes with the death of a baby but this is a different pain, when babies are assumed" (Midwife 7).

Acknowledging this similarity meant some midwives made mementos like those prepared and given to mothers with stillborn babies. Taking photos, hand and foot-prints, hair-locks, and giving baby quilts were all used to create positive memories and acknowledge the birth of the baby. The midwives hoped this may help the woman with her loss:

So they took a lot of photos of the baby, photos on her tummy. They did all these things that she had not had before (previous removal of baby) which I think really helped her even though the baby was going; it helped her, she felt a little bit better about it (Midwife 2).

Midwives described their reaction to being involved with an assumption of care as "hard", "horrible", "awful" and "traumatic". One of the midwives who worked consistently with these vulnerable women said: "Sometimes I just feel completely overwhelmed by it and just think I can't do this anymore, it's just like, way too hard" (Midwife 9). Another described how she felt she needed to control her emotional response at the actual time of removal to maintain a professional impression even though her feelings were quite different; "I get this intense feeling... just this gut-wrenching 
kind of feeling. I want to burst into tears and to hug her and tell her everything is going to be all right, but I can't. I know that I need to maintain a professional appearance" (Midwife 3).

In summary, working with women at risk of an assumption and/or who had experienced the removal of their baby evoked an array of emotions and reactions in midwives. These were expressed under the theme "Being in the heart space" and represented the emotional work midwives were required to do when working in the context of an assumption of care.

\section{DISCUSSION}

This study used a qualitative descriptive design to explore and describe the experience of 10 midwives from one Australian state who had experienced assumption of care of a newborn. "Being in the head space" and "Being in the heart space" described the dichotomy that midwives faced when caring for these women. On the one hand, midwives described how they needed to work within the existing legislative requirements that dictate specific systems and processes be followed in order to protect firstly the unborn child and then the newborn. On the other, they described experiencing significant emotional distress and conflict as they shifted from being woman centred ${ }^{16}$ to child focused. ${ }^{17}$

Midwives work in partnership with women guiding them through their pregnancy, supporting and assisting them through labour and birth and through the early postnatal period. The role of the midwife is to support the woman's relationship with her unborn baby and then her newborn within the broader context of the family. ${ }^{18}$ Unborn babies assessed to be at significant risk of harm cause a fundamental dilemma for the midwife. She must act in the best interests of the unborn, however during pregnancy this presents somewhat of a conundrum. Keeping the unborn baby safe means ensuring the pregnant woman has access to services and resources that will support her and enable the progression of a healthy pregnancy. Developing positive relationships with women to keep them engaged within a maternity service is important and integral to providing quality care especially 
given women can quite easily choose to not attend. The tension between the foci of child/newborn versus woman adds to the emotional work of the midwife.

Research by Hunter ${ }^{18}$ has previously demonstrated that emotional work in midwifery occurs when a midwife is challenged to move from a "with woman ideology" to a "with institution ideology". ${ }^{19}$ There are some synergies between her work and the findings of our study. The midwives in our study engaged in complex emotional work as they were required to firstly partner with a third external organisation; Community Services, and secondly to work within a framework that seemed to prioritise the baby over the woman and baby as a dyad. The midwives often experienced a sense of conflict between their expectations of Community Services, the decision making processes around assumption of care and their own beliefs of what was best for the dyad.

Working with a pregnant woman where child protection was a concern was difficult but being a witness and/or actively participating in the actual event of removal of her baby provoked serious emotional responses in the midwives. Knowing how to deal with emotions at the time and afterwards was a challenge. Distress and trauma were common concepts related by the midwives. Likewise, both Chapman ${ }^{6}$ and Wood ${ }^{4}$ have spoken of how midwives hold onto the difficult and painful memories of the events such as, removal of a newborn for many years afterwards. Midwives may only ever be involved in a small number of assumptions of care, yet these often have a profound impact.

A number of studies from social work and child protection have explored emotional labour ${ }^{20-22}$. Similar to our findings these studies have also demonstrated how these professionals struggle with the emotional impact of their work. Knowing how to deal with the expected emotional responses of families was acknowledged as part of the role of being involved with vulnerable parents and children. ${ }^{20-22}$ With experience, child protection workers deal with emotional responses, either by increasing their skills in engaging hostile or resistant families or knowing when to withdraw to a place of emotional safety. ${ }^{20}$ An extensive literature review of the skills required to work in child 
protection demonstrated that managing the emotional impact was not regularly recognised as a crucial part of the process. ${ }^{23}$ This supports the experiences of the midwives in our study who agreed that the level of emotional work required was not addressed in the child protection training they had attended.

Once a baby was removed, midwives equated their emotional reactions or feelings to that of caring for a woman after a stillbirth. Wood ${ }^{4}$ also identified that midwives regularly used this analogy to describe their experiences of removal of a newborn and the feeling of grief and stress. Research investigating midwives experiences of stillbirth can provide insight into how midwives can deal with their emotions during the assumption of care. Kenworthy's ${ }^{24}$ study of the experiences of midwives involved in stillbirths resonates with the midwives in our study. For example the vividness of the memories created and the extent of the emotional labour and professional grief ${ }^{25}$ matched the assumption of care experiences in our study.

While our qualitative study provides insights into this important area of practice, there were limitations. The number of participants was small and all were predominantly from metropolitan areas. These midwives' experiences may be different to those working in smaller communities, especially where they know the woman or family outside the hospital. In addition only midwives were interviewed and thus only their perspective was presented. Future work would benefit from collecting and triangulating data from a number of different key stakeholders.

Legislation in each state and territory also varies, meaning the processes and legal requirements for midwives and others differ. International laws also have different ways of determining risk of significant harm for newborn infants and children, prevention and support systems, ideologies and cultural concerns related to child protection. ${ }^{26}$ Studies in other contexts could produce helpful information for practice. 
It was beyond the scope of this study to specifically address the removal of children from Australian Indigenous families, Aboriginal and Torres Strait Islander peoples. The history of the 'stolen generation' and the profound subsequent intergenerational impacts mean Indigenous children remain over-represented in the child protection system. ${ }^{26,27}$

\section{CONCLUSION}

This study addresses a gap in the literature about how midwives experience an assumption of care of a newborn. Midwives have a unique role to play in terms of child protection. By the very nature of their work, they have responsibility for supporting the woman to grow a healthy baby and prepare for birth and the transition to motherhood. In essence to protect an unborn baby at risk means working in a positive way with the woman to ensure she remains engaged with services and able to access appropriate resources. Midwives need to be provided with support to ensure that they are able to effectively care for these women and for themselves. 


\section{Acknowledgements}

We thank the midwives who willingly and honestly participated in this study, sharing stories that were difficult and often very sad. We thank the Australian College of Midwives (NSW Branch) for financial support in the form of a scholarship, to undertake the study.

\section{Conflict of Interest}

We not have any conflicts of interest to declare 


\section{REFERENCES}

1. NSW Health. Assumption of Care Order by Community Services on Health Premises. 2011; (PD2011_065): 1-12.

2. NSW Department of Community Services. New South Wales Interagency Guidelines for Child Protection Intervention. 2006.

3. Bull L. Child protection in midwifery: A case series. Journal of Neonatal Nursing. 2008; 14: 149-55.

4. Wood G. Taking the baby away. Removing babies at birth for safeguarding and child protection. MIDIRS Midwifery Digest. 2008; 18(3): 311-9.

5. Chapman T. Safeguarding the welfare of children: 1. British Journal of Midwifery. 2002; 10(9): 569-72.

6. Chapman T. Safeguarding the welfare of children: part two. British Journal of Midwifery. 2003; 11(2): 116-9.

7. Sandelowski M. Whatever Happened to Qualitative Description. Research in Nursing and Health. 2000; 23: 334-40.

8. Sandelowski M. What's in a name? Qualitative description revisited. Research in Nursing \& Health. 2010; 33(1): 77-84.

9. Marshall C, Rossman GB. Designing Qualitative Research. California: Sage; 2006.

10. Higginbottom G. Sampling issues in qualitative research. Nurse Researcher. 2004; 12(1): 719.

11. Daly J, Willis K, Small R, Green J, Welch N, Kealy M, et al. A hierarchy of evidence for assessing qualitative health research. Journal of Clinical Epidemiology. 2007; 60(1): 43-9.

12. Braun V, Clarke V. Using thematic analysis in psychology. Qualitative Research in Psychology. 2006; 3: 77-101.

13. Burns N, Grove S. The Practice of Nursing Research: Conduct, Critique and Utilization. 5th ed. St Louis: Elsevier Sanders; 2005.

14. Jootun D, McGhee G, Marland GR. Reflexivity: promoting rigour in qualitative research. Nursing Standard. 2009; 23(23): 42-6.

15. Annells $M$, Whitehead D. Analysing data in qualitative research. In: Schneider Z, Whitehead D, Elliott D, Lobiondo-Wood G, Haber J, editors. Nursing and midwifery research: Methods and appraisal for evidenced-based practice. Third ed. Sydney, Australia: Elsevier; 2007. p. 138-55.

16. Leap N. Woman-centred or women-centred care: does it matter? British Journal of Midwifery. 2009; 17(1): 12-6. 
17. Thompson FE. The Practice Setting: Site of Ethical Conflict for Some Mothers and Midwives. Nursing Ethics. 2003; 10(6): 588-601.

18. Homer CSE, Passant L, Brodie PM, Kildea S, Leap N, Pincombe J, et al. The role of the midwife in Australia: views of women and midwives. Midwifery. 2009; 25(6): 673-81.

19. Hunter B. Conflicting ideologies as a source of emotion work in midwifery. Midwifery. 2004; 20: 261-72.

20. Leeson C. The emotional labour of caring about looked-after children. Child \& Family Social Work. 2012; 15(4): 483-91.

21. Gray B. Emotional labour and befriending in family support and child protection in Tower Hamlets. Child \& Family Social Work. 2002; 7(1): 13.

22. Reder $\mathrm{P}$, Duncan $\mathrm{S}$. Understanding communication in child protection networks. Child Abuse Review. 2003; 12(2): 82-100.

23. Keys M. Determining the skills for child protection practice: emerging from the quagmire! Child Abuse Review. 2009; 18(5): 316-32.

24. Kenworthy D. The impact of loss on midwives: Stillbirth the lived experience. Unpublished: University of Bradford; 2004.

25. Kenworthy D, Kirkham M. Midwives Coping with Loss and Grief: Stillbirth, professional and personal losses. London, UK: Radcliffe Publishing; 2011.

26. Kojan BH, Lonne B. A comparison of systems and outcomes for safeguarding children in Australia and Norway. Child \& Family Social Work. 2012; 17(1): 96-107.

27. Zhou A, Chilvers M. Infants in Australian Out-of-Home Care. British Journal of Social Work. 2010; 40(1): 26-43. 